\title{
Potencial de los aceites esenciales en el control de Phytophthora cinnamomi Rands y Fusarium sp. in vitro en Cinnamomum verum Potential of essential oils in the control of Phytophthora cinnamomi Rands and Fusarium sp. in vitro in Cinnamomum verum
}

\author{
Petra Andrade-Hoyos ${ }^{1(\mathbb{1}}$, José Alberto Urrieta-Velázquez ${ }^{(\circledR)}$, Nadia Landero-Valenzuela ${ }^{3}$, \\ Homero Reyes-de la Cruz ${ }^{(1)}$, Salvador Sampayo-Maldonado ${ }^{(\mathbb{1})}$ y Alfonso Luna-Cruz ${ }^{6:}$
}

\footnotetext{
${ }^{1}$ Instituto Nacional de Investigaciones Forestales Agrícolas y Pecuarias (INIFAP). Campo Experimental Zacatepec. Carretera Zacatepec-Galeana s/n, km 0.5, Colonia IMMS. 62780 Zacatepec, Morelos, México.

${ }^{2}$ Agricultura Protegida. CENID-RASPA, INIFAP. Margen Derecho Canal de Sacramento km 6.5., ejido Las Huertas. 35140 Gómez Palacio, Durango, México. ${ }^{3}$ Centro de Investigación en Química Aplicada. Departamento de Biociencias y Agrotecnología. Enrique Reyna H. no. 140., San José de los Cerritos. 25294 Saltillo, Coahuila, México.

${ }^{4}$ Universidad Michoacana de San Nicolás de Hidalgo. Instituto de Investigaciones Químico-Biológicas. Av. Francisco J. Múgica s/n, Ciudad Universitaria. 58030 Morelia, Michoacán, México.

${ }^{5}$ Unidad de Biotecnología y Prototipos, Facultad de Estudios Superiores Iztacala, UNAM. Los Reyes Iztacala. 54090 Tlalnepantla de Baz, Edo. de México, México. ${ }^{6}$ CONACYT-Universidad Michoacana de San Nicolás de Hidalgo. Instituto de Investigaciones Químico-Biológicas. Av. Francisco J. Múgica s/n, Ciudad Universitaria. 58030 Morelia, Michoacán, México.

‡Autor para correspondencia (alunacr@conacyt.mx)
}

\section{RESUMEN}

La canela (Cinnamomum sp.) es una especie con algunas limitantes sanitarias pero significativas, siendo Phytophthora cinnamomi y Fusarium sp. las de mayor importancia que afectan la raíz. Su control con fungicidas sintéticos ha favorecido el desarrollo de resistencia debido a su mal manejo, por lo que su control con aceites esenciales es una alternativa viable. El objetivo fue determinar el potencial antifúngico de los aceites esenciales de tomillo y clavo para el control de P. cinnamomi y Fusarium sp. aislados de Cinnamomum verum. Se evaluaron concentraciones de 60, 120 y $300 \mu \mathrm{L} \mathrm{L}^{-1}$ para los dos aceites esenciales. Los datos registrados sobre la tasa de crecimiento radial micelial de los patógenos después de la incubación de Fusarium sp. a $25 \pm 1{ }^{\circ} \mathrm{C}$ y $28 \pm 1{ }^{\circ} \mathrm{C}$ tuvo diferencia significativa en las concentraciones ensayadas. P. cinnamomi, no creció radialmente con la concentración de $300 \mu \mathrm{L} \mathrm{L}^{-1}$, mientras que con $120 \mu \mathrm{L} \mathrm{L}^{-1}$ la tasa de crecimiento fue de $0.06 \mathrm{~mm}$ por día. En tanto, con Fusarium sp. se inhibe por completo el crecimiento micelial con las dosis de 120 y $300 \mu \mathrm{L} \mathrm{L}^{-1}$ de aceites esenciales.
Con el aceite de tomillo se observó una tendencia similar en las concentraciones 120 y $300 \mu \mathrm{L} \mathrm{L}^{-1}$ con mayor efecto de inhibición de los dos patógenos. La dosis de $300 \mu \mathrm{L} \mathrm{L}^{-1}$ de los dos aceites esenciales son capaces de inhibir en su totalidad $(P \leq 0.05)$ el crecimiento de ambos patógenos. En una concentración máxima de 280 esporas de ambos patógenos; se observó que, el número de esporas se reduce hasta 73 esporas con $300 \mu \mathrm{L} \mathrm{L}^{-1}$ de aceite de tomillo. Por tanto, pueden ser una alternativa preventiva en el control de enfermedades de la raíz en canela.

Palabras clave: aceites esenciales, canela, control alternativo, Fusarium sp., P. cinnamomi.

\section{SUMMARY}

Cinnamon (Cinnamomum sp.) is a species with few but significant health limitations, of which Phytophthora cinnamomi and Fusarium sp. are the most important ones that affect the root. Their control based on synthetic fungicides has favored resistance due to poor handling, so their control with essential

Cita recomendada:

Andrade-Hoyos, P., Urrieta-Velázquez, J. A., Landero-Valenzuela, N., Reyes-de la Cruz, H., Sampayo-Maldonado, S. y Luna-Cruz, A. (2022). Potencial de los aceites esenciales en el control de Phytophthora cinnamomi Rands y Fusarium sp. in vitro en Cinnamomum verum. Terra Latinoamericana, 40, 1-10. e1004. https://doi.org/10.28940/terra.v40i0.1004 
oils is a viable alternative. The objective of this study is to determine the antifungal potential of thyme and clove - essential oils for the control of P. cinnamomi and Fusarium sp. isolated from Cinnamomum verum. Concentrations of 60, 120 and $300 \mu \mathrm{L} \mathrm{L}^{-1}$ were evaluated for both oils and phytopathogens. Data recorded on the mycelial growth rate of pathogens after incubation of Fusarium sp. at $25 \pm 1{ }^{\circ} \mathrm{C}$ and $28 \pm 1^{\circ} \mathrm{C}$ had a significant difference in the concentrations tested. When clove essential oil was used in P. cinnamomi, the pathogen did not grow with the concentration of $300 \mu \mathrm{L} \mathrm{L}^{-1}$; with $120 \mu \mathrm{L} \mathrm{L}^{-1}$ growth rate was $0.06 \mathrm{~mm}$ per day, and with the concentration of $60 \mu \mathrm{L} \mathrm{L}^{-1}$, it reached a growth rate of $1.16 \mathrm{~mm}$ per day. With respect to Fusarium sp. mycelial growth was completely inhibited with doses of 120 and $300 \mu \mathrm{L} \mathrm{L}^{-1}$ of essential oils; with thyme oil a similar trend was observed at concentrations of 120 and $300 \mu \mathrm{L} \mathrm{L}^{-1}$ greatly inhibiting effects of the two pathogens. It is noteworthy that the doses of $300 \mu \mathrm{L} \mathrm{L}^{-1}$ of the two essential oils are able to inhibit in their entirety $(P \leq 0.05)$ growth of both pathogens. The use of thyme and clove essential oils can be an alternative in preventive control of cinnamon root diseases.

Index words: essential oils, cinnamon verum, alternative control, Fusarium sp., P. cinnamomi.

\section{INTRODUCCIÓN}

Los aceites esenciales tienen potencial natural en los mecanismos de defensa de los vegetales, son compuestos volátiles producidos por muchas especies y actúan contra microorganismos fitopatógenos (Elshafie, et al., 2015). Su uso surge como una alternativa para prevenir y controlar enfermedades en la agricultura actual, cuyas prácticas de cultivo permiten utilizar determinadas sustancias en el manejo de patógenos (Robu, Covaci y Popescu, 2015). Entre los grupos importantes de plantas, las hierbas aromáticas contienen diferentes sustancias utilizadas en la medicina y agricultura para prevenir y tratar diferentes enfermedades (Juárez-Rosete et al., 2013; Chouhan, Sharma y Guleria, 2017).

El combate de enfermedades de la raíz de diversas especies de plantas cultivadas puede realizarse con aceites esenciales, cuyas propiedades con efecto fungicida (Wilson, Ghaouth y Wisniewski, 1999), pueden inhibir el crecimiento de fitopatógenos. Por otro lado, su uso y aplicación son prácticas sostenibles que mejoran la inocuidad de los cultivos y se consideran de bajo riesgo para el ser humano (Isman, 2000; VillaMartínez et al., 2015). Dicho potencial antifúngico ha incrementado el interés para prevenir enfermedades de humanos y vegetales (Celis-Forero, Mendoza y Pachón, 2012; O’Bryan, Pendleton, Grandall y Ricke, 2016; Gogoi, Baruah y Nath, 1997; Wilson et al., 1999).

Un ejemplo de ello es el eugenol contenido en el clavo aromático, cuyos efectos son similares al fenol, considerado un anestésico que a dosis altas puede causar quemaduras a la superficie de estructuras de organismos plaga (Isaacs, 1983), por ejemplo, el sitio blanco del eugenol es la membrana citoplasmática de las bacterias (Devi, Nisha, Sakthivel y Pandian, 2010). Específicamente, el monoterpeno eugenol actúa sobre Botrytis cinerea, Monilinia fructigena, Penicillium italicum, Penicillium expansum y Phytophthora citrophthora en naranjas, peras y manzanas (Amiri, Dugas, Pichot y Bompeix, 2008; Landero et al., 2016).

Otra sustancia reportada por Matiz, Fuentes y León (2015), es el aceite de tomillo, al actuar como antibacterial en el control del acné. Al respecto, Coy y Acosta (2013) mencionan que la actividad antibacteriana se debe al 1,8-cineol con $21.5 \%$ en el control de Staphylococcus aureus, mientras que en otras investigaciones se observó efectividad sobre bacterias gram positivas y negativas (Nevas, Korhonen, Lindström, Turkki y Korkeala, 2004). Este compuesto también ha mostrado cierto control sobre hongos patógenos como Botrytis cinerea, Rhizopus stolonifer y de los pseudohongos Phytophthora italicum y $P$. citrophthora en post-cosecha (Camele et al., 2010).

Por otro lado, la canela (Cinnamomum sp.) es una especie que tiene algunas limitantes sanitarias pero significativas, como el daño causado por Phytophthora cinnamomi y Fusarium sp., que el manejo que se realiza generalmente mediante fungicidas químicos, lo cual ha tenido huella ambiental, siendo su mal uso la generación de resistencia en los patógenos del suelo (Baraldi et al., 2003). Por ello, existe la necesidad de alternativas menos tóxicas, de menor impacto para el ambiente y que sean efectivas para el manejo de estas enfermedades, sugiriendo así los aceites esenciales y extractos naturales en el manejo potencial de la marchitez causada por diferentes especies de Fusarium (Abdel-Monahim, Abo-Elyousr y Morsy, 2011).

Basado en la eficacia y las diferentes propiedades de los aceites naturales de plantas aromáticas 
o medicinales, se planteó determinar el potencial antifúngico de los aceites esenciales de tomillo y clavo sobre el control in vitro de P. cinnamomi y Fusarium sp. aislados de Cinnamomum verum.

\section{MATERIALES Y MÉTODOS}

\section{Recolección de Muestras y Ubicación del Estudio}

El oomicete Phytophthora cinnamomi Rands y el hongo Fusarium sp. se aislaron de raíces de plantas de canela (Cinnamomum verum) con lesiones rojizas inducidas por Fusarium sp. y pudrición de raíces por P. cinnamomi. Durante la primavera/verano de 2018, se colectaron raíces de plantas de canela a $30 \mathrm{~cm}$ de profundidad, en Zozocolco de Hidalgo, Veracruz,

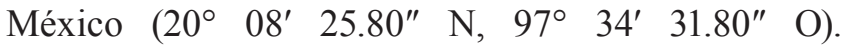
Posteriormente, las raíces se trasladaron en bolsas Ziploc $^{\circledR}$ y se procesaron en el laboratorio de hongos y patología vegetal del Centro de Agroecología del Instituto de Ciencias de la Benemérita Universidad Autónoma de Puebla.

\section{Aislamiento de Patógenos}

El oomicete $P$. cinnamomi y el hongo Fusarium sp., se aislaron de raíces de canela, de forma aleatoria, se obtuvieron fracciones de raíces de un centímetro de longitud, posteriormente, se lavaron con $\mathrm{NaClO}$ al $1 \%$ $\mathrm{v} / \mathrm{v}$ por 1 minuto y se enjuagaron tres veces (durante 3 min) con agua destilada. Para aislar a P. cinnamomi, se siguió la metodología de Andrade-Hoyos et al. (2012); los fragmentos de raíz se colocaron en cajas de Petri (de $9 \mathrm{~cm}$ ), utilizando dos medios de cultivo, uno fue papa dextrosa agar (PDA de Bioxon $^{\circledR}$ ) y jugo-V8 ${ }^{\circledR}$, para este último se adicionaron antibióticos

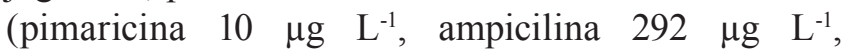
rifampicina $10 \mu \mathrm{g} \mathrm{L}^{-1}$, pentacloronitrobenceno $0.10 \mathrm{~g} \mathrm{~L}^{-1}$ e himexazol $\left.0.25 \mu \mathrm{g} \mathrm{L}^{-1}\right)$; finalmente, se colocaron a $28 \pm 2{ }^{\circ} \mathrm{C}$ por $72 \mathrm{~h}$. Para el aislamiento y purificación de Fusarium sp., se llevó a cabo un proceso similar, la siembra se hizo solo en PDA sin antibióticos y se incubó a $28 \pm 2{ }^{\circ} \mathrm{C}$ por 72 horas.

\section{Caracterización Morfológica}

La identificación morfológica del género Fusarium sp. causante de infección de raíces de canela, previamente se realizó inoculación en plántulas y se encontró la reproducción de síntomas y reaislamiento, se realizó de acuerdo con Burgess, Summerell, Bullock, Gott y Backhouse (1994) y P. cinnamomi, se llevaron a cabo estudios detallados sobre morfología de colonias, esporangios elipsoides papilados, y clamidosporas en placas de jugo V-8 clarificado, incubados a $28^{\circ} \mathrm{C}$ en la oscuridad durante 15 días. Se caracterizó con base en la metodología propuesta por Erwin y Ribeiro (1996).

\section{Aceites Esenciales}

Las muestras frescas de hojas y tallo de tomillo se recolectaron en Atlixco, Puebla, y en el mercado local se compró el clavo seco, las fechas en las que se realizaron las colectas fueron en mayo del 2018. El material vegetal se trasladó dentro de una hielera y en el laboratorio se mantuvieron a $4{ }^{\circ} \mathrm{C}$, posteriormente se realizó la extracción del aceite esencial a partir de $1 \mathrm{~kg}$ de material vegetal.

Los aceites de tomillo y clavo se extrajeron mediante hidrodestilación como lo indican Wang, Wang y Yang (2009), utilizando un equipo Clevenger ${ }^{\circledR}$, pesando $50 \mathrm{~g}$ de cada una de las especies (muestra fresca de tomillo y seca de clavo). Posteriormente, se agregó agua destilada estéril hasta cubrir en su totalidad a cada una de las muestras, enseguida se sometieron a temperatura de $100{ }^{\circ} \mathrm{C}$ por $2 \mathrm{~h}$. Los aceites se separaron del hidrolato correspondiente y se almacenaron a $4{ }^{\circ} \mathrm{C}$, previo a su uso.

\section{Control in vitro de Phytophthora cinnamomi y Fusarium sp. con Syzygium aromaticum y Thymus vulgaris}

Para la preparación de cada tratamiento con sus respectivas repeticiones, previo a la solidificación (entre 40 y $50{ }^{\circ} \mathrm{C}$ ) del medio, en placas con $100 \mathrm{~mL}$ del cultivo PDA se adicionaron los aceites Syzygium aromaticum (clavo) y Thymus vulgaris (tomillo) a tres concentraciones $\left(60,120\right.$ y $\left.300 \mu \mathrm{L} \mathrm{L}^{-1}\right)$ y cinco repeticiones por tratamiento y un tratamiento negativo (sin aceite). Posteriormente, un disco de $5 \mathrm{~mm}$ de diámetro de PDA con cultivo puro de $72 \mathrm{~h}$ de crecimiento, de P. cinnamomi y Fusarium sp., se transfirió al centro de otras placas con papa dextrosa agar (PDA) gelificado e incubó a $28 \pm 2{ }^{\circ} \mathrm{C}$ por $192 \mathrm{~h}$ (Figura 1). 


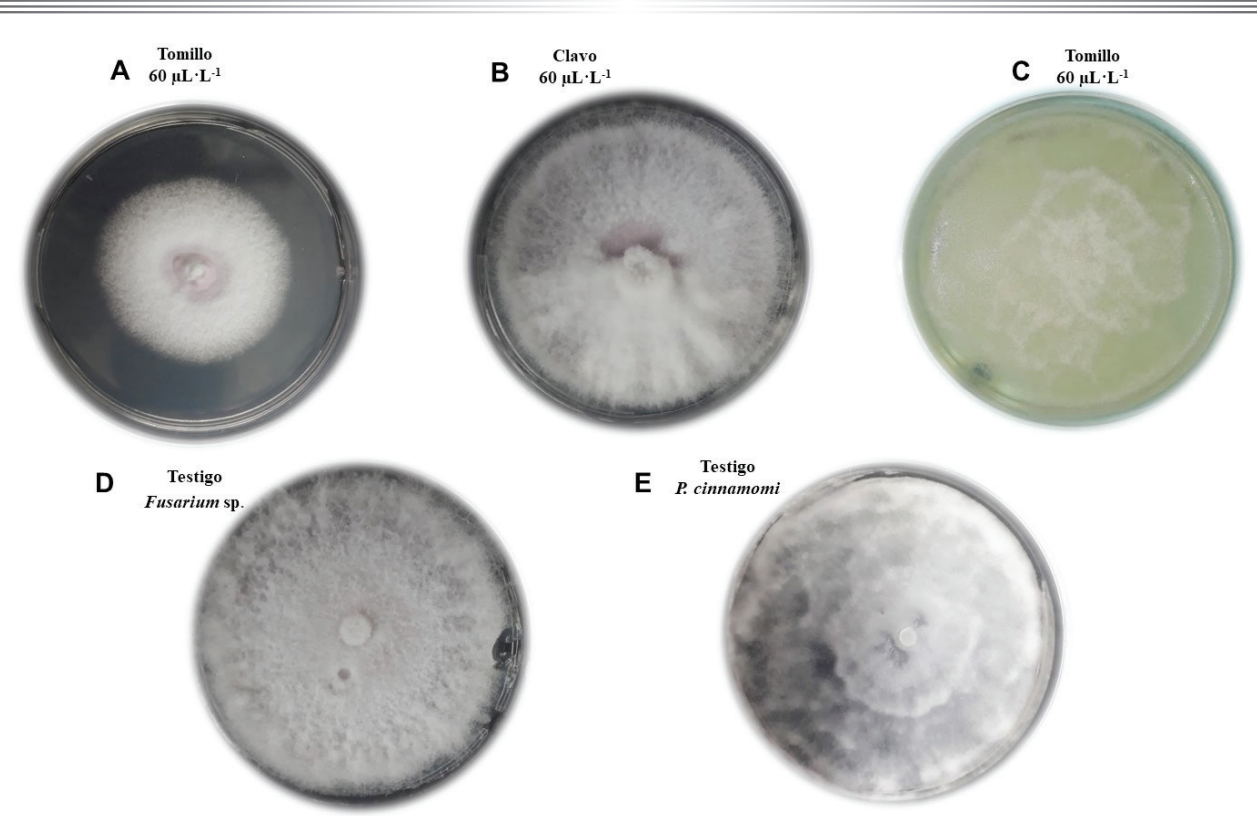

Figura 1. A, B) Crecimiento radial de Fusarium sp. en medio PDA con $60 \mu \mathrm{L} \mathrm{L}^{-1}$ de aceites esenciales de tomillo y clavo; C) P. cinnamomi en medio PDA con $60 \mu \mathrm{L} \mathrm{L}^{-1}$ de aceite de tomillo; D) testigo para Fusarium sp. y E) testigo de P. cinnamomi. Durante ocho días de crecimiento. Figure 1. A, B) Radial growth of Fusarium sp. in PDA medium with $60 \mu \mathrm{L} \mathrm{L}^{-1}$ of thyme and clove essential oils; C) P. cinnamomi in PDA medium with $60 \mu \mathrm{L} \mathrm{L}^{-1}$ of thyme oil; D) control for Fusarium sp. and E) P. cinnamomi control. During eight days of growth.

\section{Tasa de Crecimiento (Tc) de Especies Fúngicas}

Se calculó la tasa de crecimiento por día (mm/día), de acuerdo con el procedimiento establecido por Mead, Hudson y Hinton (1993), mediante la Ecuación 1. Para ello, se registraron los radios de crecimiento micelial de la colonia fúngica del día inicial y final. La Tc micelial se determinó registrando medidas con un vernier digital de los dos patógenos cada 8 h (Fusarium sp.) y 12 h (P. cinnamomi) durante 10 días (Abdel-Fattah, Shabana, Ismail y Rashad, 2007).

$\mathrm{Tc}=\frac{(\mathrm{cf}-\mathrm{ci})}{(\mathrm{Tf}-\mathrm{Ti})}$

donde:

$\mathrm{Tc}=$ tasa de crecimiento

$\mathrm{Cf}=$ crecimiento diametral final en $\mathrm{cm}$

$\mathrm{Ci}=$ crecimiento diametral inicial (día uno) en $\mathrm{cm}$

$\mathrm{Tf}=$ tiempo final del crecimiento fúngico (día final)

$\mathrm{Ti}=$ tiempo inicial (día uno)

\section{Esporulación}

La esporulación de las colonias de P. cinnamomi se cuantificó a los 19 días de incubadas y en Fusarium sp. los conidios se contabilizaron a los 15 días, para ello, cada placa de Petri se enjuagó con $15 \mathrm{~mL}$ de agua destilada estéril, la superficie se raspó ligeramente con una espátula estéril y se filtró con gasa de algodón estéril $\left(\right.$ Protec $\left.^{\circledR}\right)$. Posteriormente, se tomó una alícuota de $0.5 \mathrm{~mL}$ de la suspensión de esporas del patógeno de cada tratamiento, y se transfirieron a una cámara de Neubauer para el conteo de conidios (Landero et al., 2016).

\section{Análisis Estadístico}

El análisis de los resultados obtenidos se realizó considerando un diseño experimental completamente al azar, se realizaron análisis de regresión para los tratamientos $S$. aromaticum y $T$. vulgaris generando una ecuación con una curva representativa de cada uno de ellos (Liengme, 2002). Los resultados de crecimiento 
micelial y tasa de crecimiento de los patógenos se compararon mediante análisis de varianza utilizando un diseño factorial $2 \times 2$ y pruebas de separación múltiple de medias de Tukey $(\alpha=0.05)$ con el software SAS v.9 para Windows ${ }^{\circledR}$ (SAS, 1999). Los valores de crecimiento micelial mínimos y de producción de esporas máximos se estimaron a partir de la ecuación de regresión generada por el modelo con la ayuda del software Microsoft Excel ${ }^{\circledR}$.

\section{RESULTADOS Y DISCUSIÓN}

\section{Control in vitro de P. cinnamomi y Fusarium sp.}

Al utilizar los aceites de tomillo y clavo a concentraciones diferentes, es notorio el efecto sobre el crecimiento de los patógenos aislados de Cinnamomum (Figura 1). El análisis del crecimiento de $P$. cinnamomi y Fusarium sp. con tratamientos de aceite esencial de tomillo y clavo, mostró coeficientes de variación entre 2 y $74 \%$. Se identificaron diferencias significativas $(P \leq 0.05)$ en los efectos principales y la interacción simple en el crecimiento del micelio de ambos patógenos. Ambos aceites esenciales, a dosis bajas, muestran resultados estadísticamente iguales a los de los testigos, a excepción del aceite de tomillo que fue similar a las dosis más elevadas en control del crecimiento de los patógenos, mientras que la dosis media $\left(120 \mu \mathrm{L} \mathrm{L} \mathrm{L}^{-1}\right)$ y alta $\left(300 \mu \mathrm{L} \mathrm{L}^{-1}\right)$, inhibieron el crecimiento de Fusarium sp. y P. cinnamomi (Figura 2 y 3). Esto ha quedado de manifiesto con otros autores, quienes han investigado el potencial de estos aceites para el control de Rhizoctonia solani y Penicillium expansum en poscosecha de frutos (Landero et al., 2016), y en la inhibición del crecimiento de Rhizoctonia solani y Streptomycetes sp. (Arici y Sanli, 2014). Otro efecto observado, es la disminución de la actividad de enzimas degradadoras de la pared celular en la aplicación foliar de mentol sobre enfermedades causadas por patógenos necrótrofos en el frijol (Khaledi, Taheri y Tarighi, 2015). En el presente estudio, los aceites esenciales de clavo y tomillo tuvieron un $100 \%$ de control de Fusarium sp. y P. cinnamomi como se muestra en la Figura 2, demostrando el potencial control o inhibición de hongos fitopatógenos in vitro.

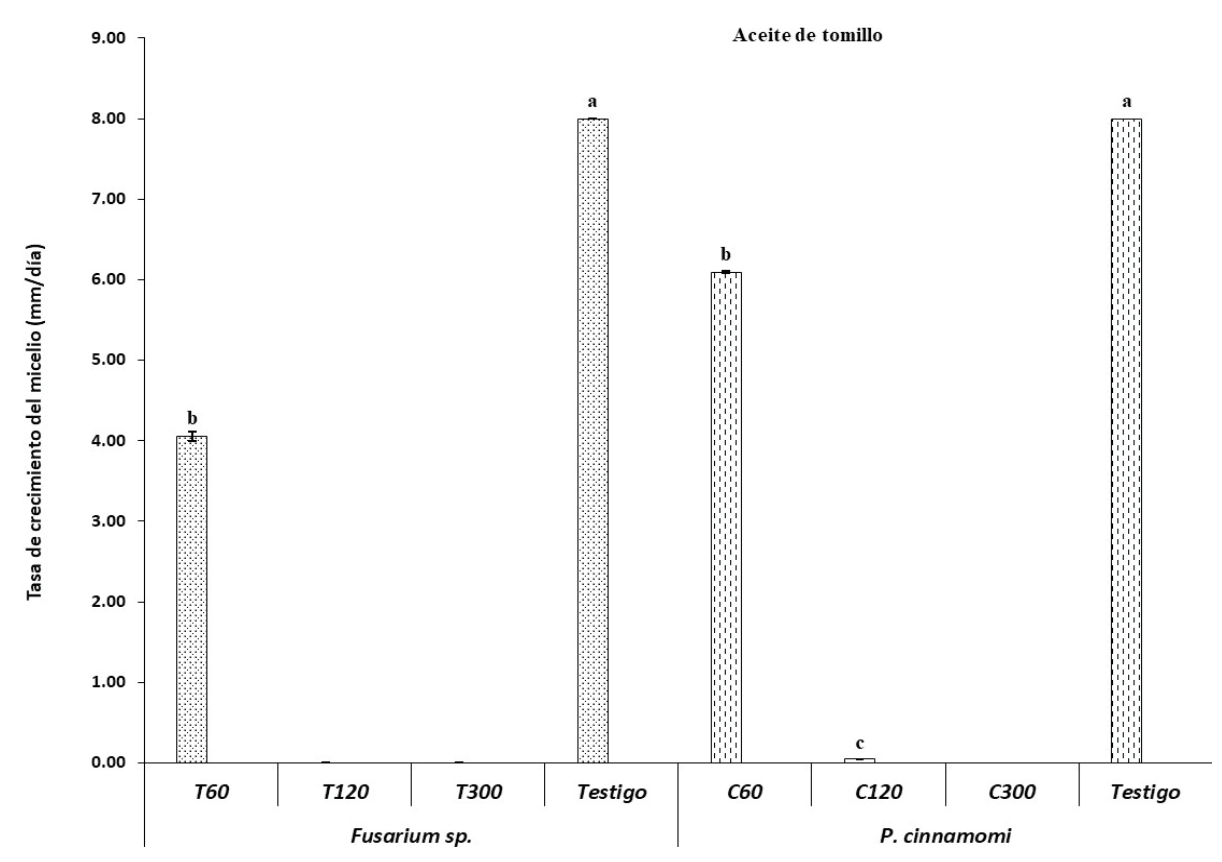

Figura 2. Crecimiento micelial in vitro de los patógenos $P$. cinnamomi y Fusarium sp. con tres dosis $\left(60,120\right.$ y $\left.300 \mu \mathbf{L ~ L}^{-1}\right)$ de aceite de tomillo (T). Barras con la mima letra son estadísticamente iguales (Tukey, $P=0.05$ ).

Figure 2. In vitro mycelial growth of the pathogens $P$. cinnamomi and Fusarium sp. with three doses $\left(60,120\right.$ and $\left.300 \mu \mathrm{L} \mathrm{L}^{-1}\right)$ of thyme oil (T). Bars with the same letter are statistically equal (Tukey, $P=0.05$ ). 
De acuerdo con el análisis de varianza (Figura 1 y 2), existen diferencias estadísticas significativas $(P \leq 0.0001)$ entre tratamientos $\left(60,120\right.$ y $\left.300 \mu \mathrm{L} \mathrm{L}^{-1}\right)$ a base de aceite esencial de clavo y tomillo sobre el crecimiento in vitro de cada uno de los patógenos. La tasa de crecimiento micelial es inversamente proporcional a las concentraciones de los aceites esenciales empleados. De tal modo que los testigos, tanto de Fusarium sp. como de $P$. cinnamomi, tuvieron una tasa de crecimiento de $8 \mathrm{~mm}$ por día. Las tres concentraciones de aceite de tomillo mostraron diferencia significativa, siendo las mejores 120 y $300 \mu \mathrm{L} \mathrm{L}^{-1}$. Sin embargo, también la dosis de $60 \mu \mathrm{L} \mathrm{L}^{-1}$, resultó mejor que el testigo (Figura 2).

Con el aceite de clavo, el crecimiento de Fusarium sp. fue nulo al utilizar dosis de 120 y $300 \mu \mathrm{L} \mathrm{L}^{-1}$, mientras que, con el mismo aceite el crecimiento de $P$. cinnamomi presentó una Tc micelial de $1.16 \mathrm{~mm}$ por día utilizando una dosis de $60 \mu \mathrm{L} \mathrm{L}^{-1}$. De igual manera, las dosis de 120 y $300 \mu \mathrm{L} \mathrm{L}^{-1}$ inhibieron la Tc micelial a un valor cercano a cero, con respecto al testigo (Figura 3).
Los aceites esenciales han demostrado potencial antimicrobiano como conservadores en el control de Rhizoctonia solani y Streptomycetes scabies en cultivo de papa (Arici y Sanli, 2014); en otros resultados, el aceite esencial de Cymbopogon citratus reduce hasta en un $60 \%$ el efecto de $P$. capsici y P. melonis (Amini, Farhang, Javadi y Nazemi, 2016). En el caso particular del aceite esencial de tomillo, se encontró que el efecto de su fase volátil es capaz de inhibir completamente el crecimiento de $P$. infestans (Soylu, Soylu y Kurt, 2006) y de la germinación de esporangios y zoosporas, y crecimiento micelial de $P$. capsici (Bi, Jiang, Hausbeck y Hao, 2012); así como en algunas especies de hongos en cereales (Matusinskya, Zouhar, Pavela y Novy, 2015). En otro estudio, los autores reportan que el follaje de orégano, romero y lavanda inhiben el crecimiento de Botritys cinerea, esto en función de la dosis utilizada (Soylu et al., 2006).

La inhibición in vitro de P. cinnamomi y Fusarium sp. con los aceites de clavo y tomillo demuestran su capacidad antimicrobiana para el control de estas dos enfermedades importantes en la producción agrícola.

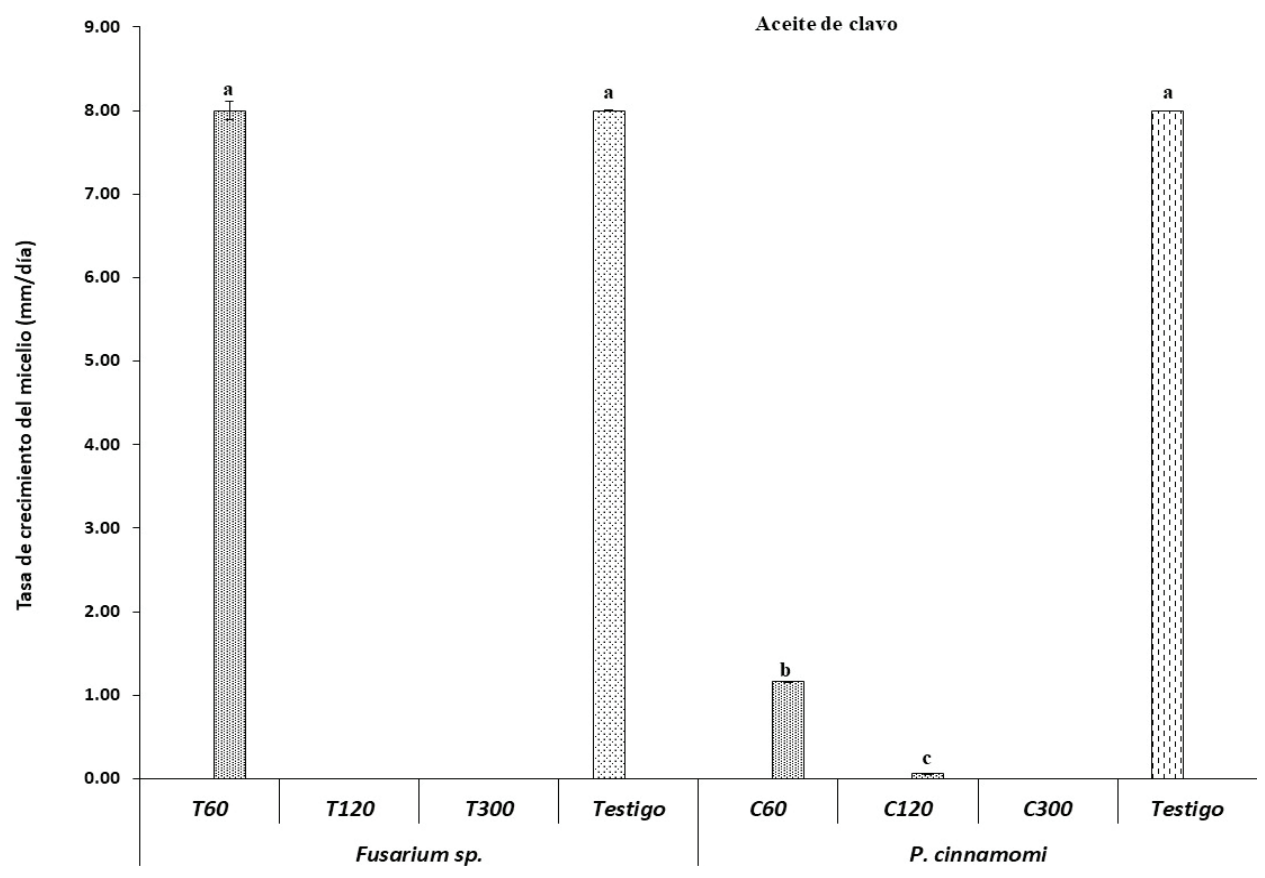

Figura 3. Crecimiento micelial en el control in vitro de los patógenos P. cinnamomi y Fusarium sp. con tres dosis de aceite de clavo $\left(\mathbf{C 6 0}, \mathbf{C 1 2 0}\right.$ y $\left.\mathbf{C 3 0 0} \mu \mathrm{L} \mathrm{L}^{-1}\right)$. Barras con la misma letra son estadísticamente iguales (Tukey, $P=0.05$ ).

Figure 3. Mycelial growth in the in vitro control of the pathogens $P$. cinnamomi and Fusarium sp. with three doses of clove oil $\left(\mathbf{C 6 0}, \mathbf{C 1 2 0}\right.$ and $\left.\mathbf{C 3 0 0} \mu \mathbf{L ~ L}^{-1}\right)$. Bars with the same letter are statistically the same (Tukey, $P=0.05$ ). 
Dicho efecto sobre el crecimiento de los hongos fitopatógenos puede atribuirse a compuestos antimicrobianos como el carvacrol y eugenol, identificados en tomillo y clavo respectivamente (Daferera, Ziogas y Polissiou, 2003). En un estudio a diferentes concentraciones de aceite esencial de canela $\left(60,120\right.$ y $\left.300 \mu \mathrm{L} \mathrm{L}^{-1}\right)$ sobre frutos de pera en poscosecha afectados por $P$. expansum, el crecimiento micelial del patógeno se detuvo $81 \mathrm{~h}$ posteriores al tratamiento con la dosis más alta (Landero et al., 2016). Por otro lado, Sharma, Rajendran, Srivastava, Sharma y Kundu (2017), encontraron que el aceite de clavo inhibió el crecimiento del micelio de Fusarium oxysporum f. sp. lycopersici 1322 y la germinación de las esporas a $125 \mu \mathrm{L} \mathrm{L}^{-1}$. Por lo tanto, sugieren que el aceite de clavo es un potente agente antifúngico que podría usarse como biofungicida para el control de $F$. oxysporum y otros patógenos de forma preventiva. Se recomienda realizar pruebas a nivel de campo, ya que existen otros factores bióticos y abióticos que disminuyen la efectividad de los aceites esenciales en el control de enfermedades en los cultivos.

\section{Esporulación}

Con el aceite esencial del tomillo, Fusarium sp. disminuyó el número de esporas producidas. De acuerdo con los modelos matemáticos, se estima que el valor máximo de producción (245 esporas por $\mathrm{mL}$ ) se presentaría si el hongo se desarrollara a una concentración de $79 \mu \mathrm{L} \mathrm{L}^{-1}$ de aceite esencial o bien, si se incrementa la concentración de este aceite arriba de $100 \mu \mathrm{L} \mathrm{L}^{-1}$, el número de esporas se reduciría completamente con $346.97 \mu \mathrm{L} \mathrm{L}^{-1}$ (Figura 3).

El modelo matemático (Figura 4), estimó la producción máxima de esporas, correspondiendo a 207 esporas por $\mathrm{mL}$ cuando el hongo se colocó a $60 \mu \mathrm{L} \mathrm{L}^{-1}$ de aceite esencial de clavo. También se observó que, por arriba de $50 \mu \mathrm{L} \mathrm{L}^{-1}$ de aceite de tomillo, el número de esporas se reduce hasta 73 esporas con $300 \mu \mathrm{L} \mathrm{L}^{-1}$. Sin embargo, después de $300 \mu \mathrm{L} \mathrm{L}^{-1}$ empieza a incrementar la cantidad de esporas (Figura 4).

La inhibición in vitro de $P$. cinnamomi y Fusarium sp. con los aceites esenciales de clavo y tomillo, indican su capacidad antimicrobiana para

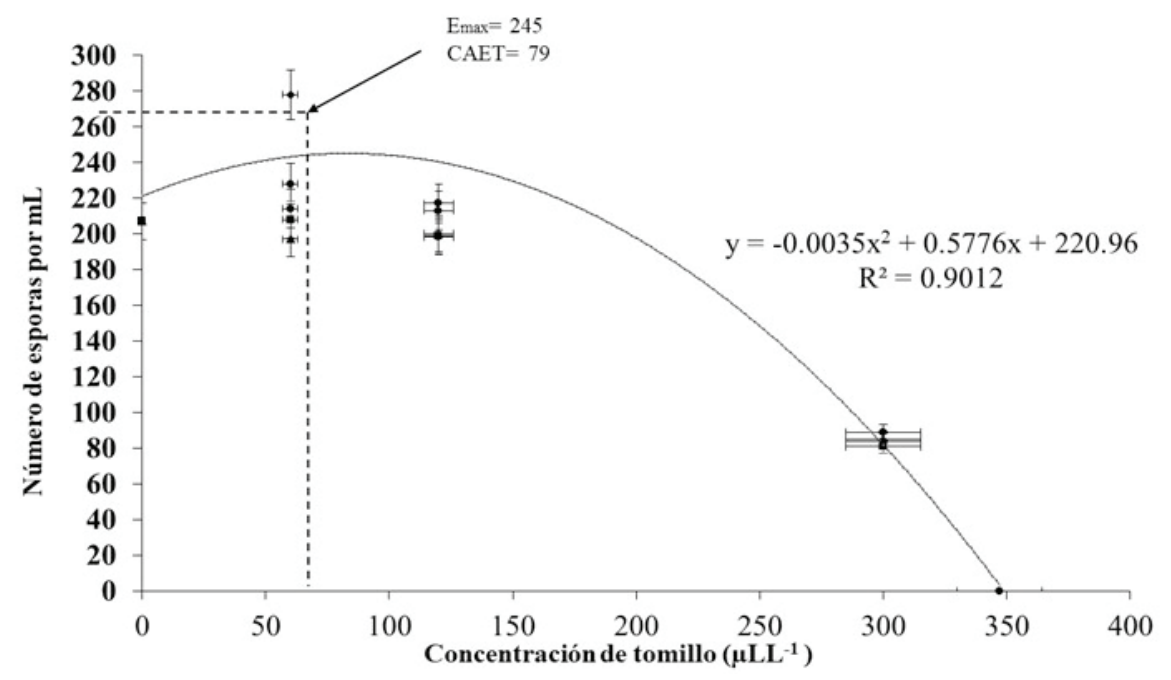

Figura 4. Producción de esporas en Fusarium sp. con aceite esencial de tomillo. Emax = producción máxima de esporas por mililitro; CAET = concentración de aceite esencial de tomillo en $\boldsymbol{\mu} \mathbf{L} \mathbf{L}^{-1}$. Los dos parámetros se estimaron a partir de la ecuación de la forma $\mathrm{y}=\mathrm{ax}^{2}+\mathrm{bx}+\mathrm{c} ; \mathrm{y}=$ número de esporas por milílitro, $\mathrm{x}=$ concentración de aceite esencial de tomillo.

Figure 4. Spore production in Fusarium sp. with thyme essential oil. Emax = maximum spore production per milliliter; CAET $=$ concentration of thyme essential oil in $\boldsymbol{\mu} \mathbf{L} \mathbf{L}^{-1}$. The two parameters were estimated from the equation of the form $y=a^{2}+$ $\mathrm{bx}+\mathrm{c} ; \mathrm{y}=$ number of spores per milliliter, $\mathrm{x}=$ concentration of thyme essential oil. 
el control de estas dos enfermedades importantes en la producción agrícola. De acuerdo con Daferera et al. (2003), el tomillo es una planta rica en carvacrol y el tomillo en eugenol; compuestos capaces de inhibir el crecimiento de algunos hongos y pseudohongos fitopatógenos (Botrytis cinerea, Fusarium sp.). Además, se ha reportado que los aceites esenciales disminuyen la severidad de algunas enfermedades afectando la germinación de esporas de algunas especies de hongos, entre ellos, Rhizopus stolonifer y Fusarium solani; esto los convierte en candidatos para la prevención de fitopatógenos en los programas de manejo integrado (Raymaekers, Ponet, Holtappels, Berckmans y Cammue, 2020).

\section{CONCLUSIONES}

Los aceites de tomillo y clavo inhibieron el crecimiento micelial de P. cinnamomi y Fusarium sp. in vitro a dosis de 120 y $300 \mu \mathrm{L} \mathrm{L}^{-1}$. El más efectivo fue el aceite de clavo al inhibir completamente el crecimiento micelial y la germinación de esporas a $120 \mu \mathrm{L} \mathrm{L}^{-1}$ con un valor $\mathrm{IC}_{50}$ de 18.2 y $0.3 \mathrm{mg} \mathrm{L}^{-1}$ respectivamente. Cabe resaltar que con $120 \mu \mathrm{L} \mathrm{L}^{-1}$ de los aceites de clavo y tomillo son suficientes para detener el crecimiento de P. cinnamomi y Fusarium sp.

Los aceites esenciales son una alternativa potencial para el control de estos dos patógenos en plantas de canela. Sin embargo, es conveniente realizar pruebas de efectividad biológica en campo para tener mayor precisión en el control de los patógenos $P$. cinnamomi y Fusarium sp.

\section{DECLARACIÓN DE ÉTICA}

No aplica.

\section{CONSENTIMIENTO PARA PUBLICACIÓN}

No aplica.

\section{DISPONIBILIDAD DE DATOS}

El conjunto de datos utilizados o analizados durante el estudio actual, están disponibles con el autor de correspondencia.

\section{CONFLICTO DE INTERESES}

Los autores declaran que no tienen intereses en competencia.

\section{FONDOS}

No hubo fondos de financiamiento público o privado para la realización de este estudio y todos los gastos corrieron a cargo de los autores.

\section{CONTRIBUCIÓN DE LOS AUTORES}

Conceptualización: P.A.H. y N.L.V. Metodología y adquisición de fondos: P.A.H., N.L.V. Análisis formal, redacción, revisión y edición: P.A.H., A.L.C., J.A.U.V., N.L.V., H.R.C., S.S.M. Supervisión: P.A.H. y A.L.C. Todos los autores leyeron y aprobaron el manuscrito final.

\section{AGRADECIMIENTOS}

Se agradece por las facilidades de trabajo en las instalaciones del laboratorio de hongos y patología vegetal ubicado en el Centro de Agroecología, Instituto de Ciencias, Benemérita Universidad Autónoma de Puebla.

\section{LITERATURA CITADA}

Abdel-Fattah, G. M., Shabana, Y. M., Ismail, A. E., \& Rashad, Y. M. (2007). Trichoderma harzianum: a biocontrol agent against Bipolaris orizae. Mycophatologia, 164, 81-89. https:// doi.org/10.1007/s11046-007-9032-9

Abdel-Monahim, M. F., Abo-Elyousr, K. A. M., \& Morsy, K. M. (2011). Effectiveness of plant extracts on suppression of damping-off and wilt diseases of lupine (Lupinus termis Forsik). Crop Protection. 30(2), 185-191. https://doi. org/10.1016/j.cropro.2010.09.016

Amini, J., Farhang, V., Javadi, T., \& Nazemi, J. (2016). Antifungal effect of plant essential oils on controlling Phytophthora species. Plant Pathology Journal, 32(1), 16-24. https://doi. org/10.5423/PPJ.OA.05.2015.0091

Amiri, A, Dugas, R., Pichot, A. L., \& Bompeix, G. (2008). In vitro and in vivo activity of eugenol oil (Eugenia caryophylata) against four important postharvest apple pathogens. International Journal of Food Microbiology, 126(1-2), 13-9. https://doi.org/10.1016/j.ijfoodmicro.2008.04.022 
Andrade-Hoyos, P., de León, C., Espíndola B., M. C., Alvarado R., D., López J., A., \& García E., R. (2012). Selección de porta-injertos de aguacate para tolerancia-resistencia a Phytophthora cinnamomi Rands. usando temperaturas controladas. Spanish Journal of Rural Development, 3(4), 2330.

Arici S. E., \& Sanli, A. (2014). Effect of some essential oils against Rhizoctonia solani and Streptomycetes scabies on potato plants in field conditions. Annual Research \& Review in Biology, 4(12), 2027-2036. https://doi.org/10.9734/ ARRB/2014/8526

Baraldi, E., Mari, M., Chierici, E., Pondrelli, M., Bertolini, P., \& Pratella, G. C. (2003). Studies on thiabendazole resistance of Penicillium expansum of pears: pathogenic fitness and genetic characterization. Plant Pathology, 52(3), 362-370. https://doi.org/10.1046/j.1365-3059.2003.00861.x

Bi, Y., Jiang, H., Hausbeck, M. K., \& Hao, J. J. (2012). Inhibitory effects of essential oils for controlling Phytophthora capsici. Plant Disease, 96(6), 797-803. https://doi.org/10.1094/PDIS11-11-0933

Burgess, L. W., Summerell, B. A., Bullock, S., Gott, K. P., \& Backhouse, D. (1994). Laboratory manual for Fusarium Research. (3 $3^{\text {rd }}$ ed.). Sydney, Australia: University of Sydney.

Camele, I., De Feo, V., Altieri, L., Mancini, E., De Martino, L., \& Rana, G. L. (2010). An attempt of postharvest orange fruit rot control using essential oils from Mediterranean plants. Journal of Medicinal Food, 13(6), 1515-1523. https://doi. org/10.1089/jmf.2009.0285

Celis-Forero, A., Mendoza-Forero, C., \& Pachón-Suárez, M. E. (2012). Plantas aromáticas silvestres promisorias por su contenido de aceites esenciales ( $1^{\mathrm{a}}$ ed.). Colombia: Produmedios.

Chouhan, S., Sharma, K., \& Guleria, S. (2017). Antimicrobial activity of some essential oils-present status and future perspectives. Medicines, 4(3), 58. https://doi.org/10.3390/ medicines 4030058

Coy B., C. A., \& Acosta, G. E. (2013). Antibacterial activity and chemical composition of essential oils of rosemary (Rosmarinus officinalis), thyme (Thymus vulgaris) and turmeric (Curcuma longa) from Colombia. Revista Cubana de Plantas Medicinales, 18(2), 237-246.

Daferera, D. J., Ziogas, B. N., \& Polissiou, M. G. (2003). The effectiveness of plant essential oils on the growth of Botrytis cinerea, Fusarium sp. and Clavibacter michiganensis subsp. michiganensis. Crop Protection, 22(1), 39-44. https://doi. org/10.1016/S0261-2194(02)00095-9

Devi, K. P., Nisha, S. A., Sakthivel, R., \& Pandian, S. K. (2010). Eugenol (an essential oil of clove) acts as an antibacterial agent against Salmonella typhi by disrupting the cellular membrane. Journal of Ethnopharmacology, 130(1), 107-115. https://doi.org/10.1016/j.jep.2010.04.025

Elshafie, H. S., Mancini, E., Sakr, S., De Martino, L., Mattia, C. A., De Feo, V., \& Camele, I. (2015). Antifungal activity of some constituents of Origanum vulgare L. essential oil against post-harvest disease of peach fruit. Journal of Medicinal Food, 18(8), 929-934. https://doi.org/10.1089/jmf.2014.0167

Erwin, D. C., \& Ribeiro, O. K. (1996). Phytophthora diseases worldwide. The Journal of Agricultural Science, 131(2), 245249. https://doi.org/10.1017/S001447979825109X
Gogoi, P., Baruah, P., \& Nath, S. C. (1997). Antifungal activity of the essential oil of Litsea cubeba. Journal of Essential Oils Research, 9(2), 213-215. https://doi.org/10.1080/10412905.199 7.9699462

Isaacs, G. (1983). Permanent local anesthesia and anhydrosis after clove oil spillage. The Lancet, 321(8329), 882. https://doi. org/10.1016/S0140-6736(83)91428-9

Isman, M. B. (2000). Plant essential oils for pest and disease management. Crop Protection, 19(8-10), 603-608. https://doi. org/10.1016/S0261-2194(00)00079-X

Juárez-Rosete, C. R., Aguilar-Castillo, J. A., Juárez-Rosete, M. E., Bugarín-Montoya, R., Juárez-López, P., \& Cruz Crespo, E. (2013). Hierbas aromáticas y medicinales en México: tradición e innovación. Revista Bio Ciencias, 2(3), 119-129. https://doi.org/10.15741/revbio.02.03.06

Khaledi, N., Taheri, P., \& Tarighi, S. (2015). Antifungal activity of various essential oils against Rhizoctonia solani and Macrophomina phaseolina as major bean pathogens. Journal of Applied Microbiology, 118(3), 704-717. https://doi. org/10.1111/jam.12730

Landero V., N., Lara V., F. M., Aguado R., G. J., Andrade H., P., Encarnación A., D., \& Pérez-R., Y. (2016). Essential oil of Cynnamomum zeylanicum: control alternative for Penicillium expansum on pear in postharvest. Revista Mexicana de Ciencias Agrícolas, 7(5), 1017-1028.

Liengme, B. V. (2002). A guide to microsoft excel 2002 for scientists and engineers. England: Newnes. ISBN13: 9780750656139

Matiz, G. E., Fuentes, K., \& León, G. (2015). Microencapsulación de aceite esencial de tomillo (Thymus vulgaris) en matrices poliméricas de almidón de ñame (Dioscorea rotundata) modificado. Revista Colombiana de Ciencias Químico Farmacéuticas, 44(2), 189-207. https://doi.org/10.15446/ rcciquifa.v44n2.56293

Matusinsky, P., Zouhar, M., Pavela, R., \& Novy, P. (2015). Antifungal effect of five essential oils against important pathogenic fungi of cereals. Industrial Crops and Products, 67, 208-215. https://doi.org/10.1016/j.indcrop.2015.01.022

Mead, G. C., Hudson, W. R., \& Hinton, M. H. (1993). Microbiological survey of five poultry processing plants in the UK. British Poultry Science, 34(3), 497-503. https://doi. org/10.1080/00071669308417605

Nevas, M., Korhonen, A. R., Lindström, M., Turkki, P., \& Korkeala, H. (2004). Antibacterial efficiency of finnish spice essential oils against pathogenic and spoilage bacteria. Journal of Food Protection, 67(1), 199-202. https://doi.org/10.4315/0362028X-67.1.199

O’Bryan, C. A., Pendleton, S. J., Grandall, P. G., \& Ricke, S. C. (2016). Potential of plant essential oils and their components in animal agriculture-in vitro studies on antibacterial mode action. Frontiers in Veterinary Science, 2, 1-8. https://doi. org/10.3389/fvets.2015.00035

Raymaekers, K., Ponet, L., Holtappels, D., Berckmans, B., \& Cammue, B. P. A. (2020). Screening for novel biocontrol agents applicable in plant disease management-A review. Biological Control, 144, 104240. https://doi.org/10.1016/j. biocontrol.2020.104240

Robu, V., Covaci, G., \& Popescu, I. M. (2015). The use of essential oils in organic farming. Research Journal of Agricultural Science, 47(4), 134-137. 
SAS Institute. (1999). SAS/STAT User guide. Release 9.0. Cary, NC, USA: SAS Institute Inc.

Sharma, A., Rajendran, S., Srivastava, A., Sharma, S., \& Kundu, B. (2017). Antifungal activities of selected essential oils against Fusarium oxysporum f. sp. lycopersici 1322, with emphasis on Syzygium aromaticum essential oil. Journal of Bioscience and Bioengineering, 123(3), 308-313. https://doi. org/10.1016/j.jbiosc.2016.09.011

Soylu, E. M., Soylu, S., \& Kurt, S. (2006). Antimicrobial activities of the essential oils of various plants against tomato late blight disease agent Phytophthora infestans. Mycopathologia, 161(2), 119-128. https://doi.org/10.1007/s11046-005-0206-Z
Villa-Martínez, A., Pérez-Leal, R., Morales- Morales, H. A., Basurto-Sotelo, M., Soto-Parra, J. M., \& Martínez-Escudero, E. (2015). Situación actual en el control de Fusarium spp. y evaluación de la actividad antifúngica de extractos vegetales. Acta Agronómica, 64(2), 194-205. https://doi.org/10.15446/ acag.v64n2.43358

Wang, R., Wang, R., \& Yang, B. (2009). Extraction of essential oils from five cinnamon leaves and identification of their volatile compound compositions. Innovative Food Science \& Emerging Technologies, 10(2), 289-292. https://doi. org/10.1016/j.ifset.2008.12.002

Wilson, C. L., Ghaouth, A. E., \& Wisniewski, M. E. (1999). Prospecting in nature's storehouse for biopesticides. Revista Mexicana de Fitopatología, 17(1), 49-53. 